\title{
A rapid and dramatic outburst in Blazar 3C 454.3 during May 2005
}

\section{Optical and infrared observations with REM and AIT}

\author{
L. Fuhrmann ${ }^{1,2}$, A. Cucchiara $^{3}$, N. Marchili ${ }^{1}$, G. Tosti ${ }^{1}$, G. Nucciarelli ${ }^{1}$, S. Ciprini ${ }^{4}, 1$, E. Molinari ${ }^{5}$, \\ G. Chincarini ${ }^{5,6}$, F. M. Zerbi ${ }^{5}$, S. Covino ${ }^{5}$, E. Pian ${ }^{7}$, E. Meurs ${ }^{8}$, V. Testa ${ }^{9}$, F. Vitali ${ }^{9}$, L. A. Antonelli ${ }^{9}$, \\ P. Conconi ${ }^{5}$, G. Cutispoto ${ }^{10}$, G. Malaspina ${ }^{5}$, L. Nicastro ${ }^{11}$, E. Palazzi ${ }^{12}$, and P. Ward ${ }^{8}$
}

1 Dipartimento di Fisica e Osservatorio Astronomico, Università di Perugia, via A. Pascoli, 06123 Perugia, Italy e-mail: lars.fuhrmann@fisica.unipg.it

2 INAF, Osservatorio Astronomico di Torino, via Osservatorio 20, 10025 Pino Torinese (TO), Italy

3 Department of Astronomy and Astrophysics, Pennsylvania State University, 525 Davey Laboratory, PA 16802, USA

4 Tuorla Astronomical Observatory, University of Turku, Väisäläntie 20, 21500 Piikkiö, Finland

5 INAF, Osservatorio Astronomico di Brera, via E. Bianchi 46, 23807 Merate (Lc), Italy

${ }^{6}$ Universitá degli studi di Milano-Bicocca, Dipartimento di Fisica, Piazza delle Scienza 3, 20126 Milan, Italy

7 INAF, Osservatorio Astronomico di Trieste, via G. B. Tiepolo 11, 34131 Trieste, Italy

8 Dunsink Observatory, Castleknock Dublin 15, Ireland

9 INAF, Osservatorio Astronomico di Roma, via Frascati 33, 00040 Monteporzio Catone, Italy

${ }^{10}$ INAF, Osservatorio Astrofisico di Catania, via S. Sofia 78, 95123 Catania, Italy

11 IASF/CNR, Sezione di Palermo, via Ugo La Malfa 153, 90146 Palermo, Italy

12 IASF/CNR, Sezione di Bologna, via Gobetti 101, 40129 Bologna, Italy

Received 28 September 2005 / Accepted 8 October 2005

\section{ABSTRACT}

The flat-spectrum radio quasar 3C 454.3 is well known to be a highly active and variable source with outbursts occurring across the whole electromagnetic spectrum over the last decades. In spring 2005, 3C 454.3 has been reported to exhibit a strong optical outburst which subsequently triggered multi-frequency observations of the source covering the radio up to $\gamma$-ray bands. Here, we present first results of our near-IR/optical ( $V, R, I, H$ band) photometry performed between May 11 and August 5, 2005 with the Rapid Eye Mount (REM) at La Silla in Chile and the Automatic Imaging Telescope (AIT) of the Perugia University Observatory. 3C 454.3 was observed during an exceptional and historical high state with a subsequent decrease in brightness over our 86 days observing period. The continuum spectral behaviour during the flaring and declining phase suggests a synchrotron peak below the near-IR band as well as a geometrical origin of the variations e.g. due to changes in the direction of forward beaming.

Key words. galaxies: active - galaxies: blazars: general - galaxies: blazars: individual: 3C 454.3 - galaxies: jets - galaxies: quasars: general

\section{Introduction}

The blazar sub-class of radio-loud Active Galactic Nuclei (AGNs) consists of flat-spectrum radio quasars (FSRQ) and BL Lacs which exhibit rather complex and outstanding characteristics such as extreme variability at all wavelengths, polarisation, strong $\gamma$-ray emission, often high superluminal motion and brightness temperatures exceeding the inverse Compton limit (e.g. Urry 1999). The overall spectral energy distribution (SED) of blazars is characterised by two broad components. The first one peaks in the IR- soft-X-ray band and is due to synchrotron emission, while the second component at higher energies is most likely produced by inverse Compton emission. The mechanism for the rapid and high amplitude variability often observed in blazars is still not well understood. Different theoretical models are discussed which include e.g. shock-in-jets (e.g. Marscher 1996), colliding relativistic plasma shells (e.g. Guetta et al. 2004) as well as changes in the direction of forward beaming (e.g. Villata \& Raiteri 1999).

The flat-spectrum radio quasar $3 \mathrm{C} 454.3(z=0.859)$ is a typical example of a highly variable blazar source. It is one of the brightest extragalactic radio sources on the sky and multi-epoch VLBI studies revealed a compact, superluminal $\left(\beta_{\text {app }} \lesssim 8 \mathrm{c}\right)$ source showing a bent, one-sided core-jet structure on mas scales (e.g. Krichbaum et al. 1995; Pauliny-Toth 1998; Pagels et al. 2004). Strong flux density variability in the radio regime was observed during long-term monitoring campaigns carried out over the last decades (e.g. Aller et al. 1997; Teraesranta et al. 1998; Ciaramella et al. 2004, and Refs. therein). While its low-frequency (decimeter) variability was partly attributed to interstellar scintillation (e.g. Altschuler et al. 1984), the source often shows strong synchrotron 


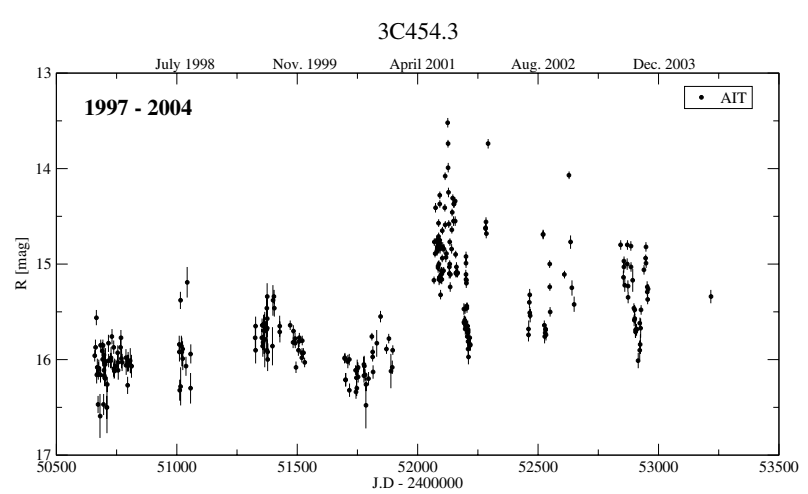

Fig. 1. Long-term light curve of 3C 454.3 obtained with the AIT telescope during a blazar monitoring project between 1997 and 2004. The source shows a very active behaviour with different variability amplitudes and time scales. The most prominent outburst occurred in August/September 2001. $R$ band data are shown as this band is most commonly observed.

outbursts on time scales of years in the $\mathrm{cm}$ - to mm-regime. These outbursts at different frequencies appear correlated often with increasing variability amplitude towards higher frequencies (e.g. Tornikoski et al. 1994). Here, a strong and consistent periodicity of about six years was found in the overall flaring behaviour of the source (Ciaramella et al. 2004). In addition, new VLBI component ejections were suggested to occur contemporaneously with radio outbursts and enhanced levels of $\gamma$-ray flux (Krichbaum et al. 1995; Pagels et al. 2004). At high energies, 3C 454.3 is a prominent example of a strong and variable source detected by e.g. Einstein, ROSAT, COMPTEL, EGRET and OSSE with a spectral maximum occurring at $\mathrm{MeV}$ energies (e.g. Blom et al. 1995; Lin et al. 1996).

In the optical band, the source was early identified as an optically violently variable. Historical $B$ band data go back to 1900 (Angione 1968) and since then several outbursts have been reported over the decades (see also Fig. 1). High optical polarisation $\left(P_{\mathrm{opt}}=0-16 \%\right)$ and polarisation angle changes were observed (see Angel \& Stockman 1980). Several attempts were made to find a correlation between the radio and optical events in 3C 454.3. Tornikoski et al. (1994) reported a possible correlation for mm-radio and optical data obtained between 1980 and 1993 confirming the previous findings of Pomphrey et al. (1976) and Balonek (1982). The latter authors suggested a correlation for the optical variations leading the radio events with a time lag of about $1.2 \mathrm{yr}$ with a tendency of considerably shorter time scales for the optical variability. In addition, intranight variability of $3 \mathrm{C} 454.3$ at optical bands was detected on several occasions ( $\sim 0.3 \mathrm{mag} / \mathrm{h}$; Angione 1971). During a monitoring campaign of $\gamma$-ray loud blazars, 3C 454.3 was found to exhibit rapid $R$ band variations of 0.3 mag in less than $3 \mathrm{~h}$ without comparable behaviour in other bands (Villata et al. 1997; Raiteri et al. 1998a).

In spring 2005 a new, exceptional strong outburst in 3C 454.3 has been reported (Balonek 2005a,b) which triggered subsequent, quasi-simultaneous broad band observations of the source at radio, IR and optical bands as well as at higher energies (X-ray $/ \gamma$-ray, Foschini et al. 2005). Here, we present first results of our multi-band photometry
( $V, R, I, H$ bands) performed during the new outburst phase between May and August 2005 probably showing the most rapid variations (and historical brightness) observed in this source so far. Comprehensive papers in preparation will present and analyse this data in the framework of a broad band spectral energy approach using the quasi-simultaneous observations at $\mathrm{cm}$-to mm-wavelengths as well as data obtained at higher energies with the SWIFT and INTEGRAL satellites (Giommi et al. 2005; Pian et al. 2005; Fuhrmann et al. 2005).

\section{Observations and data reduction}

The photometric optical/IR monitoring of 3C 454.3 started on May 11, 2005 in the $V, R, I$, and $H$ band. The last data presented here were obtained on August 5, 2005 and thus our observations cover a total time period of 86 days (JD 2453 502.4-2453 588.0). The observations were carried out with two instruments: (i) the Newtonian f/5, $0.4 \mathrm{~m}$, Automatic Imaging Telescope (AIT) of the Perugia University Observatory, Italy and (ii) the Rapid Eye Mount (REM, Zerbi et al. 2004), a robotic telescope located at the ESO Cerro La Silla observatory in Chile. The AIT is based on an equatorially mounted $0.4-\mathrm{m}$ Newtonian reflector having a $0.15-\mathrm{m}$ refractor solidly joined to it. AIT is a robotic telescope equipped with a $192 \times 165$ pixels CCD array, thermo-electrically cooled with Peltier elements and Johnson-Cousins BVRI filters are utilised for photometry (Tosti et al. 1996). The REM telescope has a Ritchey-Chretien configuration with a $60 \mathrm{~cm} \mathrm{f} / 2.2$ primary and an overall $\mathrm{f} / 8$ focal ratio in a fast moving alt-azimuth mount providing two stable Nasmyth focal stations. At one of the two foci the telescope simultaneously feeds, by means of a dichroic, two cameras: REMIR for the NIR (see Conconi et al. 2004), and ROSS (see Tosti et al. 2004) for the optical. Both cameras have a field of view of $10 \times 10 \mathrm{arcmin}$ and imaging capabilities with the usual NIR $\left(z^{\prime}, J, H\right.$ and $\left.K\right)$ and optical broad band $(V, R, I)$ filters. Moreover, via an Amici prism, low resolution slitless spectroscopy is also possible.

At the AIT telescope images were taken at $R$ and $I$ bands during a total of 30 nights, whereas $V, R, I, H$ band REM observations were performed during 23 nights of the 86 days observing period (see Table 1). The data were usually taken as a sequence of two or more frames per band and night. Each single $H$ band observation with REMIR was performed with a dithering sequence of five images shifted by a few arcsec. These images are automatically elaborated using the jitter script of the ECLIPSE package. The script aligns the images and co-adds all the frames to obtain one average image for each sequence.

After dark-, bias- and flat-field correction, each image of both telescopes was reduced using standard procedures. A combination of the DAOPHOT (Stetson 1988) and Sextractor packages (Bertin \& Arnouts 1996) was used to perform standard aperture photometry. The instrumental magnitudes were then further processed to obtain standard magnitudes of 3C 454.3 and the relevant errors through differential photometry. Here, an average zero-point correction of each optical frame was applied using the tabulated magnitudes of 5-8 standard reference stars in the comparison star sequence reported by Fiorucci et al. (1998) and Raiteri et al. (1998b). 
Table 1. A short summary of the observations for each band performed between May and August 2005. Here, $T_{\text {obs }}$ denotes the observing duration and $N_{\text {data }}$ the total number of data points obtained over this time interval.

\begin{tabular}{c|cccccc}
\hline \hline & $\begin{array}{c}T_{\text {obs }} \\
\text { [days] }\end{array}$ & $N_{\text {data }}$ & $\langle\mathrm{mag}\rangle$ & $\Delta \mathrm{mag}$ & $\begin{array}{c}\left\langle F_{v}\right\rangle \\
{[\mathrm{mJy}]}\end{array}$ & $\begin{array}{c}\Delta F_{v} \\
{[\mathrm{mJy}]}\end{array}$ \\
\hline$V$ & 60 & 48 & $14.16 \pm 0.73$ & 2.37 & $13.7 \pm 9.7$ & 36.6 \\
$R$ & 86 & 85 & $13.57 \pm 0.63$ & 2.61 & $17.5 \pm 10.0$ & 47.6 \\
$I$ & 86 & 72 & $12.90 \pm 0.65$ & 2.46 & $25.4 \pm 15.4$ & 64.6 \\
$H$ & 42 & 167 & $10.18 \pm 0.36$ & 1.60 & $92.6 \pm 30.7$ & 142.6 \\
\hline
\end{tabular}

The calibration of the NIR data was done using the comparison star sequence published by González-Pérez et al. (2001). Errors on the calibrated magnitudes of 3C 454.3 were calculated taking into account the statistical error of the reduction process (aperture photometry) plus the rms of the variations as seen in the reference star zero-points. For the subsequent spectral analysis the data sets were corrected for galactic extinction according to Schlegel et al. (1998).

The calibrated light curves of the two telescopes were then combined in the $R$ and $I$ band, respectively. Here, a comparison among the data acquired with the two different telescopes in the same night did not show significant instrumental offsets. These offsets have been computed, for each band, from data acquired contemporaneously by the two telescopes (simultaneous observations occured only in 5 nights). All values were found to lie within the individual magnitude errors and thus were negligible. The final light curves of all bands are presented in Fig. 2 while Table 1 gives a short summary of the observations and results for each band by means of average magnitudes and flux densities $F_{v}$.

\section{Results and discussion}

During the last decade 3C 454.3 passed through several active states as visible in our archival long-term light curve obtained with the AIT telescope between 1997 and 2004. In Fig. 1, $R$ band data are shown as this is the band most commonly observed. Although rather undersampled, the light curve shows long-term trends with faster and stronger outbursts superimposed. The most prominent feature is visible around August/September 2001, when the source reached a magnitude of $R=13.5$ and subsequently decreased in brightness by $\Delta R=1.7$ during a period of only about 10 days. The observations presented here show $3 \mathrm{C} 454.3$ during a new outburst phase when the source was detected at its historical maximum brightness in all bands. All light curves in Fig. 2 show 3C 454.3 in an exceptional bright state at the beginning of May 2005 with a subsequent long-term decrease over the 86 days observing period as seen in the $R$ and $I$ bands. Preliminary data of Balonek $(2005 \mathrm{a}, \mathrm{b})$ indicate that the maximum brightness ( $R \sim 12 \mathrm{mag}$ ) occurred two days before the start of our observations (May 09, 2005), thus we observed the source during the declining phase of the outburst. In addition to the longterm decrease in brightness, all bands show several faster, simultaneous flares superimposed with time scales of often only a few days. The first occurred around JD 2453510 when the

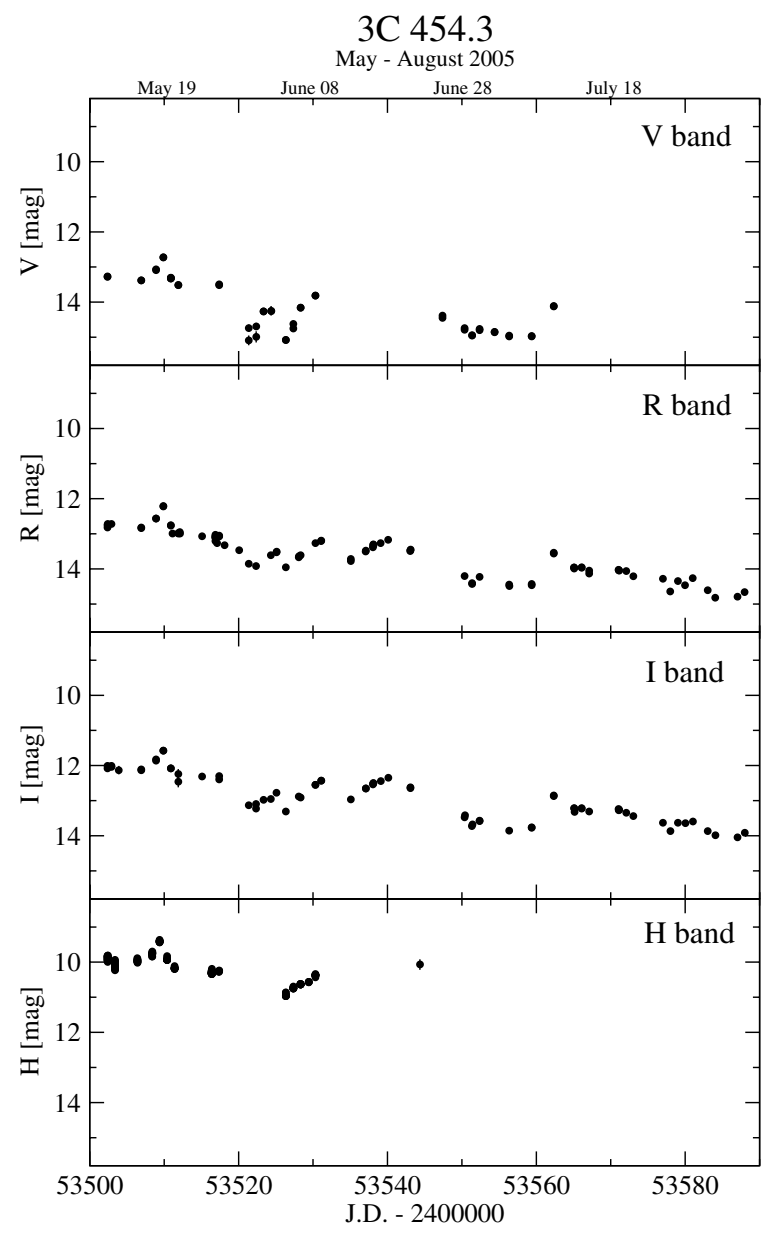

Fig. 2. Final optical and near-IR light curves of 3C 454.3 obtained between May and August 2005. The $V$ and $H$ band data cover a significantly shorter time interval. All light curves are plotted on the same scale for a better comparison. The photometric errors are often comparable to the symbol size.

source reached its maximum in all light curves with $V=12.7$, $R=12.2, I=11.6$ and $H=9.4$, respectively. The overall highest minimum-to-maximum variations are seen in the $R$ band with $\Delta R=2.6$ over a time period of 75 days (see also Table 1 ).

In Fig. 3 the continuum spectral evolution over six epochs is shown at several levels of intensity. These $V$ to $H$ band spectra were taken during periods of temporal overlap of the four bands between May 11 and June 08 (JD 2453502.42453530.3). Power-law fits to each epoch show no strong and significant spectral changes over this period. Such "achromatic" variability behaviour suggests a geometrical origin of the observed variations due to changes in the direction of forward beaming (e.g. a helical/precessing jet; Begelman et al. 1980; Villata \& Raiteri 1999) rather than acceleration/cooling mechanisms in the jet of 3C 454.3. However, the steep spectral shape with a mean value of $\alpha=1.39 \pm 0.07\left(F(v) \propto v^{-\alpha}\right)$ indicates that the maximum of the synchrotron peak was located at lower frequencies than the near-IR band during the maximum as well as the declining phase of the outburst. Here, the quasi-simultaneous observations obtained at $\mathrm{cm}$ - and mm-wavelengths will help to study the spectral shape, peak and 


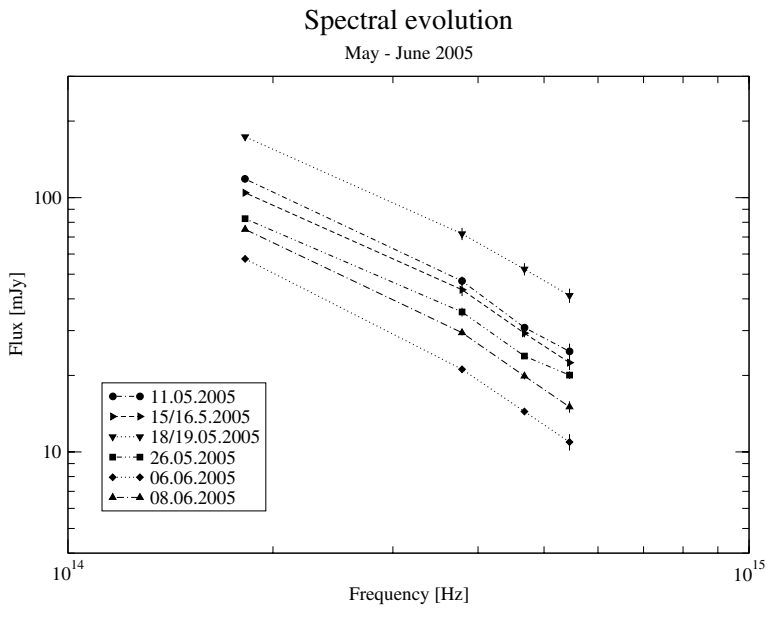

Fig. 3. Near-IR/optical continuum spectra obtained at six epochs between May 11 and June 8, 2005. Over this time period no strong, significant spectral changes are evident.

evolution of the synchrotron part of the SED of 3C 454.3 during the recent flare in more detail (Fuhrmann et al. 2005).

First results of (quasi-) simultaneous broad band observations obtained this year indicate that $3 \mathrm{C} 454.3$ was in a similar active state over the last months. During the period of our REM and AIT observations, the SWIFT satellite observed the source at three epochs between May 11 and May 19, 2005 as part of an ongoing X-ray investigation of a sample of blazar sources. First results are presented by Giommi et al. (2005) and display an exceptional, by a factor of about 10 higher X-ray flux compared to previous ROSAT observations. The simultaneous REM IR/optical and SWIFT UV/X-ray data show a complex variability and SED behaviour which requires a more detailed analysis.

\section{Summary and conclusions}

The blazar 3C 454.3 went through a dramatic, historical outburst at the beginning of May 2005. Our photometric monitoring of the source with the REM and AIT telescopes over a time period of 86 days shows a very bright state at the beginning of May 2005 with a subsequent, long-term decrease in brightness with the highest minimum-to-maximum variations of $\Delta R=2.6$. The highest brightness in our $V$ to $H$ band light curves was detected during a fast, secondary outburst occurred around May 19, 2005. A first analysis of the spectral behaviour of the source across our near-IR/optical bands during six epochs between May and June 2005 displays a steep and, in time, nearly constant spectral slope with $\langle\alpha\rangle=1.39 \pm$ 0.07 . This suggests (i) a maximum of the synchrotron emission in the SED of 3C 454.3 to be below the near-IR band in Fig. 3 and (ii) a geometrical origin of the variations. Here, changes in the viewing angle of the jet with respect to the line of sight and thus temporal changes of the Doppler factor could be invoked. However, a more detailed analysis of the synchrotron component will become possible combining our observations with (quasi-) simultaneous data obtained at radio- and mm-bands, as will be discussed in a forthcoming paper (Fuhrmann et al. 2005).
Acknowledgements. The work presented here was partly supported by the European Institutes belonging to the ENIGMA collaboration acknowledge EC funding under contract HPRN-CT-2002-00321.

\section{References}

Aller, M. F., Marscher, A. P., Hartman, R. C., et al. 1997, AIP Conf. Proc., 410, 1423

Altschuler, D. R., Broderick, J. J., Dennison, B., et al. 1984, AJ, 89, 1784

Angel, J. R. P., \& Stockman, H. S. 1980, ARA\&A, 18, 321

Angione, R. J. 1968, PASP, 80, 339

Angione, R. J. 1971, AJ, 76, 25

Balonek, T. J. 1982, Ph.D. Dissertation

Balonek, T. J. 2005a, vsnet-alert 8383

Balonek, T. J. 2005b, vsnet-alert 8405

Begelman, M. C., Blandford, R. D., \& Rees, M. J. 1980, Nature, 287, 307

Bertin, E., \& Arnouts, S. 1996, A\&AS, 117, 393

Blom, J. J., Bloemen, H., Bennett, K., et al. 1995, A\&A, 295, 330

Ciaramella, A., Bongardo, C., Aller, H. D., et al. 2004, A\&A, 419, 485

Conconi, P., Cunniffe, R., D’Alessio, F., et al. 2004, Proc. SPIE, 5492, 1602

Fiorucci, M., Tosti, G., \& Rizzi, N. 1998, PASP, 110, 105

Foschini, L., Cocco, G. Di, Malaguti, G., et al. 2005, The Astronomer's Telegram, 497, 1

Fuhrmann, et al. 2005, A\&A, in preparation

Giommi, et al. 2005, A\&A, in preparation

González-Pérez, J. N., Kidger, M. R., \& Martín-Luis, F. 2001, AJ, 122,2055

Guetta, D., Ghisellini, G., Lazzati, D., \& Celotti, A. 2004, A\&A, 421, 877

Krichbaum, T. P., Britzen, S., Standke, K. J., et al. 1995, Proceedings of the National Academy of Science, 92, 11377

Lin, Y. C., Bertsch, D. L., Dingus, B. L., et al. 1996, ApJS, 105, 331

Marscher, A. P. 1996, Energy Transport in Radio Galaxies and Quasars, ASP Conf. Ser., 100, 45

Pagels, A., Krichbaum, T. P., Graham, D. A., et al. 2004, European VLBI Network on New Developments in VLBI Science and Technology, 7

Pauliny-Toth, I. I. K. 1998, IAU Coll. 164: Radio Emission from Galactic and Extragalactic Compact Sources, ASP Conf. Ser., 144, 75

Pian, et al. 2005, A\&A, in preparation

Pomphrey, R. B., Smith, A. G., Leacock, R. J., et al. 1976, AJ, 81, 489

Raiteri, C. M., Ghisellini, G., Villata, M., et al. 1998a, A\&AS, 127, 445

Raiteri, C. M., Villata, M., Lanteri, L., Cavallone, M., \& Sobrito, G. 1998b, A\&AS, 130, 495

Schlegel, D. J., Finkbeiner, D. P., \& Davis, M. 1998, ApJ, 500, 525

Stetson, P. B. 1988, PASP, 99, 191

Teraesranta, H., Tornikoski, M., Mujunen, A., et al. 1998, A\&AS, 132, 305

Tornikoski, M., Valtaoja, E., Terasranta, H., et al. 1994, A\&A, 289, 673

Tosti, G., Pascolini, S., \& Fiorucci, M. 1996, PASP, 108, 706

Tosti, G., Bagaglia, M., Campeggi, C., et al. 2004, Proc. SPIE, 5492, 689

Urry, C. M. 1999, Astroparticle Physics, 11, 159

Villata, M., \& Raiteri, C. M. 1999, A\&A, 347, 30

Villata, M., Raiteri, C. M., Ghisellini, G., et al. 1997, A\&AS, 121, 119

Zerbi, F. M., Chincarini, G., Ghisellini, G., et al. 2004, Proc. SPIE, 5492,1590 